\title{
State-of-the-art, challenges, and future trends in security constrained optimal power flow
}

\author{
F. Capitanescu ${ }^{\mathrm{a}, *}$, J.L. Martinez Ramos ${ }^{\mathrm{b}}$, P. Panciatici ${ }^{\mathrm{c}}$, D. Kirschen ${ }^{\mathrm{d}}$, \\ A. Marano Marcolinie ${ }^{\text {, L. Platbrood }}{ }^{\mathrm{f}}$, L. Wehenkel $^{\mathrm{g}}$ \\ ${ }^{a}$ Department of electrical engineering and computer science, University of Liège, Belgium \\ ${ }^{b}$ Department of electrical engineering, University of Seville, Spain \\ ${ }^{c}$ RTE-DMA, Versailles, France \\ ${ }^{d}$ Department of electrical engineering, University of Washington, USA \\ ${ }^{e}$ Department of electrical engineering, University of Seville, Spain \\ ${ }^{f}$ Tractebel engineering, Brussels, Belgium \\ ${ }^{g}$ Department of electrical engineering and computer science, University of Liège, Belgium
}

\begin{abstract}
This paper addresses the main challenges to the security constrained optimal power flow (SCOPF) computations. We first discuss the issues related to the SCOPF problem formulation such as the use of a limited number of corrective actions in the post-contingency states and the modeling of voltage and transient stability constraints. Then we deal with the challenges to the techniques for solving the SCOPF, focusing mainly on: approaches to reduce the size of the problem by either efficiently identifying the binding contingencies and including only these contingencies in the SCOPF or by using approximate models for the post-contingency states, and the handling of discrete variables. We finally address the current trend of extending the SCOPF formulation to take into account the increasing levels of uncertainty in the operation planning. For each such topic we provide a review of the state of the art, we identify the advances that are needed, and we indicate ways to bridge the gap between the current state of the art and these needs.
\end{abstract}

\footnotetext{
* Corresponding author

Email addresses: capitane@montefiore.ulg.ac.be (F. Capitanescu), camel@us.es (J.L. Martinez Ramos), patrick.panciatici@rte-france.com (P. Panciatici), kirschen@uw.edu (D. Kirschen), alejandromm@us.es (A. Marano Marcolini), ludovic.platbrood@gdfsuez.com (L. Platbrood), 1. wehenkel@ulg.ac. be (L. Wehenkel)
} 
Keywords: mixed integer linear programming, mixed integer nonlinear programming, nonlinear programming, optimal power flow, security constrained optimal power flow

\section{Motivation}

The SCOPF $[1,2]$ is an extension of the OPF problem [3, 4] which takes into account constraints arising from the operation of the system under a set of postulated contingencies. The SCOPF problem is a nonlinear, nonconvex, large-scale optimization problem, with both continuous and discrete variables $[1,2]$. The SCOPF belongs therefore to the class of optimization problems called Mixed Integer Non-Linear Programming (MINLP).

The SCOPF has become an essential tool for many Transmission System Operators (TSOs) for the planning, operational planning, and real time operation of their system $[5,6,7,8]$. Furthermore, in several electricity markets (e.g. PJM, New-England, California, etc.) the locational marginal prices calculated using a DC SCOPF are used to price electricity. This approach is also under consideration in other systems $[9,10,11]$.

Several papers discussing the challenges to the OPF problem were published during the 90's $[5,6,7,8]$. Since then several important changes have taken place not only in power systems operation and control but also in mathematical programming:

- Power systems operate today in conditions that are more "stressed" and were not foreseen at the planning stage. In particular the increase in load has not been supported by an adequate upgrade of the generation and transmission systems. Furthermore the creation of electricity markets has led to the trading of significant amounts of electrical energy over long distances.

- These operating conditions are also more uncertain, because of the development of distributed generation based on renewable sources, and the introduction of intra-day electricity markets.

- To counteract these trends an increasing number of complex control devices (e.g. HVDC links, and FACTS devices) is being installed.

- Consequently, it can be argued that the level of security of power systems has been weakened to the point where in some systems the "N-1" 
security criterion cannot be met without resorting to corrective actions. This is partly due to market pressures which discourage from interfering in the market and thus favour the use of corrective control over preventive rescheduling. Furthermore, the trend towards smarter grid operation requires satisfying the "just-in-time" control principle according to which (day-ahead planned) preventive control is no longer sustainable while interruptible load curtailment becomes a more important option of corrective control [9].

- Day-ahead operational planning by the TSOs has become an exercise in managing uncertainty in which the SCOPF plays an important role.

- Optimization theory has progressed significantly since the 90's and the performance of solver engines has improved considerably. In particular, mature engines, mainly based on interior-point methods are now able to solve Non-Linear Programming (NLP) problems, while the performance of Mixed Integer Linear Programming (MILP) engines has improved by several order of magnitudes.

A number of issues make the SCOPF much more challenging than the OPF problem: the significantly larger problem size, the need to handle more discrete variables describing control actions (e.g. the start up of generating units and network switching) and the variety of corrective control strategies in the post-contingency states.

These factors encouraged the authors to address the delicate problem of challenges to the SCOPF computations.

This paper is based on the work of the PEGASE project [12] which brought together SCOPF specialists from academia, software providers, and utilities. Questionnaires and interviews conducted with ten European TSOs both within and outside the PEGASE consortium have helped us to clarify and define their needs in terms of SCOPF computations [13].

The remaining of the paper is organized as follows. Section II presents the formulation of the conventional SCOPF problem and Section III discusses the challenges arising from this formulation. Section IV focuses on SCOPF solution methods, with a particular emphasis on how to reduce the size of the problem size and handle discrete variables. Section V discusses new formulations of the SCOPF problem more suitable for an uncertain environment. Section VI concludes. 


\section{Formulation of the conventional SCOPF problem}

The conventional SCOPF problem can be compactly formulated as follows [2]:

$$
\begin{array}{cll}
\underset{\mathbf{x}_{0}, \ldots, \mathbf{x}_{c}, \mathbf{u}_{0}, \ldots, \mathbf{u}_{c}}{\min } & f_{0}\left(\mathbf{x}_{0}, \mathbf{u}_{0}\right) \\
\text { s.t. } & \mathbf{g}_{0}\left(\mathbf{x}_{0}, \mathbf{u}_{0}\right)=\mathbf{0} & \\
& \mathbf{h}_{0}\left(\mathbf{x}_{0}, \mathbf{u}_{0}\right) \leq \mathbf{L}_{l} & \\
& \mathbf{g}_{k}^{s}\left(\mathbf{x}_{k}^{s}, \mathbf{u}_{0}\right)=\mathbf{0} & k=1, \ldots, c \\
& \mathbf{h}_{k}^{s}\left(\mathbf{x}_{k}^{s}, \mathbf{u}_{0}\right) \leq \mathbf{L}_{s} & k=1, \ldots, c \\
& \mathbf{g}_{k}\left(\mathbf{x}_{k}, \mathbf{u}_{k}\right)=\mathbf{0} & k=1, \ldots, c \\
& \mathbf{h}_{k}\left(\mathbf{x}_{k}, \mathbf{u}_{k}\right) \leq \mathbf{L}_{m} & k=1, \ldots, c \\
& \left|\mathbf{u}_{k}-\mathbf{u}_{0}\right| \leq \bar{\Delta}_{k} & k=1, \ldots, c
\end{array}
$$

where $f_{0}$ is the objective function, and for the $k$-th system configuration ( $k=0$ corresponds to the pre-contingency configuration, while $k=1, \ldots, c$ correspond to the $c$ post-contingency configurations), $\mathbf{x}_{k}$ is the vector of state variables (i.e. magnitude and angle of voltage at all buses), $\mathbf{x}_{k}^{s}$ is the vector of state variables in short-term time frame (i.e. before the operator has had time to modify the control variables following a contingency), $\mathbf{u}_{k}$ is the vector of control variables (e.g. generators' active power, generators' terminal voltage, ratio of controllable transformers, reactance of the shunt elements, angle of the phase shifters, status of the breakers), $\overline{\Delta \mathbf{u}}_{k}=T_{k} d \mathbf{u}_{k} / d t$ is the vector of maximal allowed adjustments of the control variables between the base case and the $k$-th post-contingency state, $T_{k}$ is the interval of time available for corrective actions to ensure the feasibility of the post-contingency state and $d \mathbf{u}_{k} / d t$ is the rate of change of the control variables in response to a contingency. Finally $\mathbf{L}_{s}, \mathbf{L}_{m}$, and $\mathbf{L}_{l}$ denote respectively the short-term (emergency) limits, the medium-term limits, and the long-term (normal) operating limits.

Constraints $(2,3),(4,5)$, and $(6,7)$ enforce the feasibility of the pre-contingency state, the short-term post-contingency state (denoted by the superscript $s$ ), and the medium-term post-contingency state, respectively. Equality constraints $(2,4,6)$ represent the $\mathrm{AC}$ power flow equations. Inequality constraints $(3,5,7)$ include physical limits on the equipments (e.g. bounds on the generators' active and reactive power output, ratio of controllable transformers, reactance of shunts) as well as operational limits on the branch currents and 
voltage magnitudes. Inequalities (8) are "coupling" constraints aimed at preventing unrealistic adjustments of the control variables between the base case and the post-contingency states.

Some TSOs define several limits for a given equipment depending on the amount of time frame during which a limit violation can be endured. These limits must satisfy $\mathbf{L}_{l} \leq \mathbf{L}_{m} \leq \mathbf{L}_{s}$. For example, there may be several limits on the current or power flowing through a branch. Indeed, three line current limits are defined in the French EHV network, with line disconnection delays of respectively 1, 5 and 20 minutes [14]. The operational rules assume that the operator is unable to react within one minute and the first limit must therefore be satisfied by preventive actions only. Furthermore, in this state the use of a typical constant power load model might be not realistic, especially if significant voltage drop took place [15]. It is expected that the operator can take a single corrective action (usually a pre-defined topology change) to satisfy the second limit within 5 minutes. Satisfying the third limit may require more corrective actions of different types, such as redispatching generation or starting up fast units.

The "preventive" SCOPF [1] (denoted hereafter PSCOPF) is a particular formulation of the SCOPF that does not consider the possibility of corrective actions in post-contingency states, other than those that take place automatically (e.g., active power of generators participating in frequency control, automatic tap-changers, capacitor and reactor bank switching, secondary voltage control, SPS). Therefore, in the PSCOPF the values of the non-automatic control variables are thus the same in all system states, i.e. $\mathbf{u}_{k}=\mathbf{u}_{0}, \forall k=1, \ldots, c$.

\section{Issues related to the SCOPF problem formulation}

\subsection{Modelling of a limited number of post-contingency control actions}

A drawback of the conventional SCOPF problem relates to the choice of the corrective actions to be used for each contingency [8, 16, 17]. Typically, system operating companies define for each contingency a list of possible corrective actions that could be taken within a given period of time after the occurrence of the contingency. Because the most efficient corrective actions for each contingency may change due to the increasing amount of day-ahead uncertainty, this approach may lead to sub-optimal results i.e. higher cost of preventive actions. 
Emulating how human operators react under such conditions is a challenge for OPF tools that has long been recognized but not yet properly solved $[5,6,8,16,18]$. Operators are able to implement only a limited number of controls actions and will typically choose the ones that they perceive as being the most "effective". However, ranking control actions is not easy because the effectiveness of an action is not necessarily related to its magnitude and because almost every control variable contributes in a non separable way to both improving the objective and satisfying the constraints [18].

A few papers have proposed techniques for limiting the number of control actions implemented by the OPF. They rely on the following approaches: prior specification of the controls participating in the OPF [5, 18], sensitivity of the objective and constraints to control actions [19], approximation of the integer constraint on the maximal number of controls allowed to move using a nonlinear constraint [20,21], mathematical programming with equilibrium constraints [21], embedding the DC approximation of the OPF in a MILP formulation and focusing on topological actions [22], and combination of first order sensitivities and MILP [23], respectively.

However, as opposed to the OPF, no results appear to have been reported on techniques to limit the number of corrective actions used in postcontingency states by a SCOPF. These approaches are designed for one system state and could possibly be extended to the SCOPF problem but it is difficult to predict how this might affect computational performance when dealing with a large number of postulated contingencies.

A rigurous way to limit the number of corrective actions in the SCOPF formulation (1)-(8) is by directly including a set of constraints limiting the number of corrective actions allowed in each post-contingency state. Conceptually this can be formulated as follows:

$$
\begin{array}{ll}
-\mathbf{s}_{k} \overline{\Delta \mathbf{u}}_{k} \leq \mathbf{u}_{k}-\mathbf{u}_{0} \leq \mathbf{s}_{k} \overline{\Delta \mathbf{u}}_{k} & k=1, \ldots, c \\
\mathbf{1}^{T} \mathbf{s}_{k} \leq N_{k} & k=1, \ldots, c \\
\mathbf{s}_{k} \in\{0,1\} & k=1, \ldots, c
\end{array}
$$

where, for contingency $k, N_{k}$ is the maximum number of corrective actions allowed and $\mathbf{s}_{k}$ is a vector of statuses of corrective actions. If the status of the corrective action $u_{k j}$ is equal to 1 (resp. 0) it means that this action is (resp. not) allowed, i.e. $-\overline{\Delta u}_{k j} \leq u_{k j}-u_{0 j} \leq \overline{\Delta u}_{k j}$ (resp. $u_{k j}=u_{0 j}$ ).

Since SCOPF computation model both very fast (or quasi-instantaneous) controls, such as load curtailment, shunt reactive power, transformer ratio, 
and comparatively slower controls, such as generation redispatch, the constraint (10) can be expressed alternatively in terms of the time needed for controls implementation (e.g. $\mathbf{s}_{k}^{T} \mathbf{t}_{k} \leq T_{k}$, where $\mathbf{t}_{k}$ is the vector of times needed to implement the control actions).

Solving the conventional SCOPF augmented with constraints (9)-(11) for several values of $N_{k}$ makes it possible to determine: (i) the best trade-off between the objective function and the number of control actions used in the optimization and (ii) to assess the degree of sub-optimality that results from a limit on the number of controls and whether there is enough room for manoeuvre in the case where some control actions would fail.

\subsubsection{Determining the minimum number of control actions}

Determining the minimum number of control actions required to rescue the system from an emergency state ${ }^{1}$ or an insecure state ${ }^{2}$ to a normal state is a very important information for system operators because they need to assess whether there is enough time to implement the planned control actions $[5,6,16,24]$.

This objective function can be expressed as:

$$
\min \mathbf{1}^{T} \mathbf{s}_{0}
$$

where $\mathbf{s}_{0}$ is a vector of statuses of preventive actions. To solve this problem the conventional SCOPF constraints (6)-(8) are augmented with the constraints:

$$
-\mathbf{s}_{0} \overline{\Delta \mathbf{u}}_{0} \leq \mathbf{u}_{0}-\overline{\mathbf{u}}_{0} \leq \mathbf{s}_{0} \overline{\Delta \mathbf{u}}_{0}, \mathbf{s}_{0} \in\{0,1\}
$$

where $\overline{\mathbf{u}}_{0}$ is the vector of current values of preventive actions. The constraints (9)-(11) may also have to be considered.

\subsubsection{Determining the sequence of control actions}

Once a limited number of corrective actions sufficient to ensure a viable state of the system after the inception of any postulated contingency has been determined, the TSOs need to know in what sequence these control actions must be taken. This information is very important to avoid exacerbating

\footnotetext{
${ }^{1}$ An emergency state is a current or predicted state where some branch current or voltage magnitude limits are severely violated.

${ }^{2} \mathrm{An}$ insecure state is a state where the available corrective actions are insufficient to secure the system with respect to some credible contingencies.
} 
already violated limits or causing significant violations of other limits [6, 24]. This requires a dynamic simulation of the sequence of control actions (see section 3.2) [25].

\subsection{Handling voltage and transient stability in SCOPF}

The preventive and corrective actions identified by the SCOPF must be validated using time-domain simulation in order to ensure that they will not cause any instability $[8,25,26]$.

An implicit assumption of the conventional SCOPF formulation is that, after the occurrence of a contingency, the system will not lose stability and (with or without post-contingency corrective actions) will reach a viable steady-state. The validity of this assumption depends on the system dynamics which are not modeled in the conventional SCOPF. Therefore, the SCOPF problem is often formulated in a conservative way by imposing strong constraints on the amount of usable post-contingency controls and the target feasible region. However, this may lead to sub-optimality and an undetected risk of instability.

Due to their different time frames transient stability and voltage stability are handled separately in the SCOPF.

Two classes of approaches to handle transient and voltage stability constraints in SCOPF can be distinguished:

- class $A$ : include stability constraints in the form of the algebraic equations stemming from the various time steps of time domain simulation. This approach provides an optimal benchmark solution but it requires onerous computations and its practical feasibility remains to be proven.

- class B: include simpler (mostly linear) heuristic stability constraints that are derived after running time domain simulation. They generally lead to a smaller and acceptable increase in the size of the SCOPF but may lead to sub-optimal solutions. These approaches generally require several iterations to refine the heuristic constraints.

Most Transient Stability Constrained Optimal Power Flow (TSCOPF) approaches belongs to the class A [27, 28, 29, 30]. Approaches belonging to class $\mathrm{B}$ rely on the single machine equivalent method and either build simple heuristic constraints $[31,32]$ or merely modify the active power limits of "critical" generators [33]. 
Furthermore, most TSCOPF methods compute preventive actions that stabilize only one contingency at a time [27, 28, 31, 32]. Fewer TSCOPF methods consider several contingencies simultaneously [29, 30].

Voltage stability constrained SCOPF approaches belonging to class A either incorporate the discretized system trajectory as constraints into the SCOPF itself $[34,35]$ or include only simpler static constraints ensuring either the existence of a post-contingency long-term equilibrium $[36,37]$, or the existence and viability of both the post-contingency long-term equilibrium after the corrective actions have act and the post-contingency short-term equilibrium before corrective actions can start e.g. in the form of constraints $(4,5)[15]$. On the other hand, approaches of class B rely on: the modification of the right-hand-side term of the coupling constraints (8) as an output of the combination between: SCOPF, post-contingency optimal power flow, and quasi-steady state time domain simulation [25], maintaining adequate reactive power reserves in the area prone to instability [38], or including in PSCOPF linear voltage stability constraints derived separately using quasisteady state dynamic simulation [39].

\section{Issues related to the SCOPF problem solution}

\subsection{Reducing the size of the SCOPF problem}

\subsubsection{Motivation}

The major challenge of the SCOPF is the size of the problem, especially for large systems and caser where many contingencies are considered $[5,6,7,8]$. Trying to solve this problem directly for a large power system, by imposing simultaneously all post-contingency constraints, would lead to prohibitive memory and CPU times requirements. Moreover, because in real life applications most contingencies do not constrain the optimum, including them all into the SCOPF problem is unnecessary and may lead to numerical problems. This is especially true under stressed operating conditions, i.e. when the SCOPF is most useful.

We discuss hereafter several approaches to reduce the size of the SCOPF problem [40]. They rely on reducing the number of contingencies to be included in the SCOPF, on reducing the size of the post-contingency states, or both. 


\subsubsection{SCOPF approach using the full model for post-contingency states}

This approach essentially aims to identify efficiently an as small as possible subset including all binding contingencies (i.e. the smallest subset of the full postulated contingency set which provides the same optimal objective value as the full set) at the SCOPF optimum.

This approach iterates four modules until all post-contingency constraints are met: a SCOPF which considers only a subset of potentially binding contingencies, a steady-state security analysis (SSSA) based on a classical power flow software, a contingency filter $(\mathrm{CF})$, and a post-contingency $\mathrm{OPF}$ to check whether contingencies can be secured by corrective actions ${ }^{3}[1,41$, $42,43,44,45]$.

The performance of iterative modular SCOPF approaches strongly depend on the quality of the contingency filtering. Most $\mathrm{CF}$ techniques rank contingencies using a severity index (SI) and select those yielding a SI above some threshold $[1,5,41,42,43,46]$. These SIs are typically computed from post-contingency quantities obtained with various load flow models, except for $[44,46]$ which exploit the Lagrange multipliers of a relaxed PSCOPF solution. The main shortcomings of SI-based techniques are that parameters such as the number of top ranked contingencies selected, SI thresholds, and weights relative to different types of constraint violations require tuning. Another CF approach that avoids these drawbacks is the non-dominated contingency (NDC) technique [44, 45]. The latter is a parameter-free technique and relies on the comparison (at each iteration of the SCOPF procedure) of the constraints violations between postulated contingencies. The NDC approach generally outperforms SI-based techniques in the context of both SCOPF [45] and PSCOPF [44], providing slightly better results for the latter problem. Furthermore, the iterative SCOPF approach using NDC filtering technique has generally outperformed the Benders decomposition approach [45].

Note that if the size of the SCOPF problem after including the full model of binding contingencies exceeds the available computer memory ${ }^{4}$, this ap-

\footnotetext{
${ }^{3}$ This is due to the power flow program in the SSSA does not take into account some time-varying control actions (e.g., generation rescheduling, shunt reactance change, etc.) to check whether the violated constraints can be removed by corrective actions. This module is not needed in the particular case of the PSCOPF, where the SSSA decides whether the current optimal base case is the optimum of the SCOPF.

${ }^{4}$ In the PEGASE project the size of the European EHV system to be optimized is about
} 
proach cannot be applied. One therefore has to rely on approaches using simplified models for contingency constraints, as explained hereafter.

\subsubsection{SCOPF approaches using simplified models for post-contingency states}

Benders decomposition approach. Since the seminal paper [2], generalized Benders Decomposition (BD) [48, 49] has been widely used to solve various SCOPF problems for optimizing of operational cost, reactive power planning, computation of available transfer capability, and other power system issues $[2,36,45,50,51,52,53,54,55,56,57,58]$. The SCOPF problem is considered either as such $[2,36,45,50,51,52,53,54,55]$, or is embedded in a more general formulation such as unit commitment $[56,57,58]$. In the context of the unit commitment problem most SCOPF approaches use simplified linear (dc) formulation, in order to reduce problem complexity, except for [57].

In the $\mathrm{BD}$ approach the original SCOPF problem is decomposed into a master problem and several slave sub-problems which interact iteratively. It is very appealing due to the possibility of keeping the size of both the master and slave problems very tractable, i.e. at almost the same as optimizing a system pre- or post-contingency state only. It also makes it possible to distribute computations among several processors, which can considerably speed-up computations [2]. On the other hand, BD requires (theoretically) the convexity of the feasible region, which cannot be guaranteed in an ac SCOPF. Consequently BD must be used with great care $[2,51]$.

Linearization of relevant post-contingency constraints. The SCOPF problem can also be simplified by adding to the base case constraints only the relevant post-contingency inequalities, linearized around the optimized base case, while dropping all post-contingency equality constraints (which are however checked at the optimal solution) $[1,5,7,41,42,43,53,59,60]$. Furthermore, some approaches also use a linear model for the base case leading to a linearized SCOPF problem. The latter is solved by successive linear programming techniques [41]. The main advantage of LP-based methods is that their convergence to the global optimum of the linearized problem is guaranteed and very fast. Robust solvers are available for this class of optimization problems [41, 61]. In particular the dc power flow model is sometimes used for

13.000 buses and the number of "N-1" contingencies is about 30.000 [47]. The model of the North American Eastern Interconnection includes about 43.000 buses and considers about 70.000 contingencies [11]. 
the active power re-dispatch SCOPF problems [41, 62]. However, the linear approximation may be questionable under highly loaded conditions or when using "reactive power" control variables (e.g. LTC ratio, shunt reactance, generator terminal voltage).

Network compression approach. This approach relies on the "compression" of post-contingency states [63]. It consists in keeping the exact model of a "direct" area, where the contingency has a significant impact, and of an "indirect" area, comprising control variables which have an important effect on the constraints of the direct area. The external network of these two areas is then compressed using a REI-Dimo equivalent. The compression parameter is adaptively chosen to comply with the limits on computer memory. The more contingencies one has to include in the SCOPF, the more compressed will be the post-contingency states.

Consequences of using simplified post-contingency models. When using approaches that rely on simplified post-contingency models, it is possible that, at the solution, either the objective is sub-optimal or some original postcontingency constraints are still violated. Consequently, the post-contingency constraints must be checked at the solution. If some constraints are violated to an unacceptable extent, the problem is re-solved using an improved linearizations of the violated constraints or even using the complete model of the corresponding contingencies.

\subsubsection{PEGASE approach}

Approaches using simplified post-contingency models are generally mutually exclusive but complementary with the modular approach using the full model.

When dealing with very large SCOPF problems it could be of interest, if not mandatory due to the problem size, to use jointly exact and approximate approaches to reduce the size of the problem.

For instance the approach adopted in the PEGASE project consists in using a modular iterative SCOPF approach jointly with the "network compression" technique, with the former technique looking for the subset of potentially binding contingencies, and the latter technique further compressing the size of the post-contingency states corresponding to the potentially binding contingencies [47]. 


\subsection{Efficient algorithms for continuous SCOPF relaxation solution}

When optimizing the continuous variables of the SCOPF while freezing the values of discrete variables or treating them as continuous, the SCOPF becomes a NLP problem.

NLP is an optimization field that has reached maturity over the last decade. It is commonly agreed that two main classes of methods are best suited for solving general NLP, namely: barrier methods (mainly the interior point method) and active set methods (e.g. sequential quadratic programming, trust regions). Consequently, most state of the art NLP solvers use algorithms based on either Interior Point Method (IPM) or active set sequential quadratic programming.

The advent of IPM constituted a breakthrough for the OPF solution and since the 90's it has become a standard approach to the OPF solution. IPM has been applied to a large variety of OPF problems such as: minimization of generation cost $[60,64,65]$, minimization of active power losses [64, 66], minimization of load shedding [67], computation of loadability margin [68]. The "higher-order" algorithms (e.g. the predictor-corrector [64, 66, 69] and the multiple centrality corrections [70]) exhibit the best behaviour. IPM has been applied successfully to OPF problems not only for test systems but also to models of actual systems.

On the other hand, trust regions approaches have not received much attention in the OPF context until very recently [71, 72, 73, 74]. They focus more on algorithm robustness and global convergence than on computational speed. They have been applied generally to small systems and while the results obtained are promising, these approaches clearly require further investigations.

Several generic solvers for NLP problems have reached maturity and could be used as an engine for solving the SCOPF: KNITRO [75], IPOPT [76], LOQO [77], to mention just a few.

Although several SCOPF packages are available on the market, for obvious commercial reasons but with some exceptions [41, 42, 73], the details of the algorithms that have been implemented have not been disclosed in scientific papers. Since the only information available is usually a list of the features, a rigorous comparison of these packages is not feasible and will therefore not be attempted in this paper. Furthermore, whereas the performances of generic NLP solvers can be compared directly using public test data sets of various sizes (e.g. on platforms such as GAMS or NEOS), such test data is unfortunately not available for OPF problems. 


\subsection{Handling of discrete variables}

The efficient handling of discrete variables in the OPF has been recognized as a challenging problem and has received significant attention since the late 80 's $[5,8,18,78]$. However the SCOPF problem is a very large MINLP problem. Despite the significant progress achieved recently on the development of general purpose engines to handle such problems, a rigorous solution of the SCOPF problem by means of classical MINLP methods (e.g. branch and bound, outer approximation, etc.) remains intractable for very large systems with very large number of discrete variables, especially for applications close to real-time. Therefore fast and robust heuristic techniques are needed to deal with the discrete variables in the SCOPF.

\subsubsection{Classification of discrete variables}

From the perspective of methods used to handle discrete variables which appear in the SCOPF it is convenient to divide them in two categories:

1. variables with small discrete steps (e.g. ratio of LTCs, angle of phase shifters, reactive power of some shunts);

2. variables with large discrete steps (e.g. reactive power of some shunts, binary variables representing network switching, connection of initially non-dispatched generators, connection or disconnection of shunt compensation, non-differentiable discrete piece-wise cost curves for bids in market-based OPF [74] or in market-based congestion management).

\subsubsection{Handling of discrete variables with small steps}

The simplest, fastest, and for a long time widely used approach for handling discrete variables is based on the round-off strategy. The round-off is performed in one shot [18, 78] or progressively in several steps [73]. These round-off approaches act "blindly" because they do not consider the consequences of the discretization on the objective or the constraints. They thus have two drawbacks: the feasibility of the solution is not guaranteed if no method to restore feasibility is implemented, and the objective value may be unacceptably sub-optimal.

To overcome these drawbacks a large spectrum of heuristic approaches have been proposed. These methods include the use of penalty functions within NLP or LP solvers [79, 80], ordinal optimization [81], recursive mixedinteger linear programming [82], interior point cutting plane [83, 84], consideration of the sensitivities of the objective function and inequality constraints 
with respect to discrete variables change [85], global optimization methods (e.g. genetic algorithms [86], simulated annealing [87], tabu search [88], or hybrid approaches coupling genetic algorithms and local search NLP solvers [89]).

Most of these techniques have been proposed and tested for the basic OPF problem and not for the SCOPF, where the number of discrete variables is significantly larger. Their performance on large systems in terms of robustness and computational time remains to be assessed.

\subsubsection{Handling of discrete variables with large steps}

Network switching actions. Because they have a very low cost and are generally very effective, network switching operations, such as the connection or disconnection of transmission lines and transformers, or the splitting of busbars, are often the first post-contingency corrective action to be implemented $[13,40]$.

System operators usually know which switching operation is most effective for a given overload or a voltage problem and many of these are incorporated in the written operational rules. On the other hand, considering the increasing amount of uncertainty that affects system operation, finding the sequence of switching actions that achieves the best trade-off between optimality and number of control actions is very far from being trivial.

Since the early 80's [90, 91] the use of switching actions has been extensively studied in the context of OPF for various purposes: line overload alleviation [42, 90, 91, 92, 93, 94, 95, 96, 97], undervoltages/overvoltages mitigation [94, 96, 98, 99], system security enhancement [100, 101], generation dispatch cost reduction [22, 42, 62, 101], power loss reduction [102, 103], or a combination of these objectives [62, 96, 101].

The approaches dealing with switching actions proposed in the literature focus on the reduction of the search space of possible switching actions [99]. These rely on one of the following techniques: heuristic techniques (e.g. considering as switching candidates only the lines electrically close to the overload [90]), simulating the switching by using compensated current/flow injection $[92,102]$, distribution factors to relate the flow in the switched element to the other lines [93], maximal flow - minimal cost algorithm [100], sensitivities of power flows in overloaded branches with respect to the flows on branches candidate to be opened and MILP [95], ranking of candidate switching actions according to a performance index and simulation of the top candidates in a branch and bound fashion [92, 94, 96, 98], dc power flow 
model and MILP [22, 62, 97, 101, 103], genetic algorithms [97], mathematical programming with equilibrium constraints [47], etc.

Comprehensive reviews of techniques used for dealing with switching actions until the mid 80's and the end of the 90's can be found in [104] and [99], respectively.

Very little efforts have been made to incorporate network switching in SCOPF $[42,62,101]$. These approaches use a dc power flow model. In order to limit the number of possible network configurations to be analyzed, switching actions are implemented as corrective control actions but not as preventive actions. Corrective switching as an operational rule should be properly modeled in SCOPF, so as it is activated when the monitored constraint is violated.

Connecting of initially non-dispatched units. In order to remove post-contingency violated constraints some system operators consider the start-up of fast generating units among the possible corrective actions [13, 17, 40, 105]. However, none of the existing SCOPF program reports being able to handle this type of corrective action.

Also no SCOPF formulation considers the connection of initially nondispatched units as a preventive action for instance when the SCOPF problem is infeasible with the available set of preventive and corrective actions $[13,17$, 40, 105]. This stems from the fact that in the day-ahead operational planning the SCOPF is typically performed on the basis of the generation schedule provided by a Security Constrained Unit Commitment (SCUC) program. Since most SCUC approaches include network constraints within the UC using a dc power flow model $[56,105,106]$, they can handle branch current constraints but do not address properly voltage constraints. Nevertheless, notable progress has been achieved recently by embedding an ac SCOPF model into the SCUC $[57,58]$ or coupling the linear sensitivity-based SCOPF solutions [105], to consider voltage constraints and SCOPF infeasibility due to inadequate units commitment. If the SCOPF problem is infeasible, the SCUC must be performed again to modify the initial units commitment. However, because operation uncertainty is usually not considered in the dayahead SCUC, if the system does not follow closely the forecast assumed in the SCUC, performing the SCOPF close to real time may lead to an infeasible problem unless some units are started up in order to satisfy post-contingency constraints. Since performing the SCUC close to real time is computationally onerous, the modelling of units start up as preventive action is a required 
development in SCOPF.

\subsubsection{Unified handling of both types of discrete variables}

Instead of using separate approaches for these two classes of discrete variables it is also possible to treat them in a unified way. To this end, the MILP approach applied to the linearization of either the whole SCOPF problem or each post-contingency state seems an appropriate choice, especially due to the excellent performance of MILP engines. This is a satisfactory approach when dealing with the reactive power redispatch problem to remove voltage problems. When dealing with thermal overloads in the context of optimal active power dispatch problems, the dc power flow approximation could also be used, followed however by an ac power flow check.

\section{Beyond the classical SCOPF formulations}

\subsection{Motivation}

The SCOPF tools are intended to support decision making in planning, operation planning, and operation, to determine optimal strategies ensuring that, if events with a significant probability take place, the system will continue to operate in normal conditions.

The classical SCOPF formulation described in the previous sections is a two-stage decision making problem. The first stage decisions are called preventive controls, are applied in real time on a fully known operating state, and have a direct effect on the cost function. The second stage decisions corresponds to hypothetical corrective controls which would be applied only following the occurrence of certain contingencies to ensure viability. These actions are typically modeled as "zero cost" decisions because they have to be implemented only rarely. The set of "second stages" is finite and is assumed known a priori. Its size is roughly proportional to the system size, because the classical "N-1" security criterion yields one scenario for each possible element outage.

While this classical formulation is indeed very useful, it does not cover anymore in a fully satisfactory way the needs encountered in today's operation and operational planning environments. Indeed, due to the increasing penetration of renewable and other uncontrollable generation sources, the set of contingencies should now also incorporate (possibly large) variations in power injections in addition to equipment failures. The operators and planners must anticipate second stage decisions to deal with these "injection 
pattern contingencies" which span complex continuous spaces and are highly dependent on system conditions and on real-time information gathered about exogenous variables such as weather forecasts and market prices. Furthermore, because of the geographical extension of electric power markets, the introduction of closer to real-time markets, and to avoid that control actions taken by a TSO do not harm security of other systems reducing thereby the risk of cascading events, the decisions regarding power system operation and operational planning have to take into account larger transmission networks. It is thus essential that control actions taken by one system operator do not harm the security of neighbouring systems. In very large interconnections, the probability that more than one element becomes unavailable is no longer negligible. As the various control areas of an interconnection become more closely coupled through interchanges, the risk of cascading events therefore increases and it might become necessary to consider security criteria that go beyond the classical "N-1" and develop techniques able to quickly identify harmful N-K $(\mathrm{K}>1)$ events with non negligible probabilities. Static and equally weighted contingency lists are then no longer appropriate to model uncertainty and such list should become dynamic to exploit all the information available at the moment of taking decisions. In other words, the set of relevant scenarios and the weight given to each of these scenarios when taking first-stage decisions should be optimized.

These changes suggest that a larger (in theory uncountable) contingency sets should be considered to model the uncertainty between successive decision stages. It is also very likely that a two-stage reduction of the optimization problem will no longer be sufficient. Instead, one might have to define a multistage framework, where the couplings between decisions and uncertainties induced by adverse scenarios over longer time horizons could be modeled better.

In terms of problem formulation, the above considerations lead to two complementary and intertwined directions of research. The first one focuses on the development of appropriate methods for on-line scenario (i.e. contingency) selection adapted to the optimal control problem formulations taking into account the uncertainty affecting power system operation and planning. The second aims at revising the temporal control horizon and its decomposition into successive decision making stages. These problems could be addressed in principle using to two different frameworks, namely the robust optimal control framework [107] and the multistage stochastic programming framework [108]. 
We elaborate on these frameworks below in order to identify the corresponding needs for data collection and for algorithmic developments. Then we their practical pros-and-cons for power system security management.

\subsection{Multistage decision making under uncertainty}

The general problem faced by power system engineers in charge of security management and control can be seen as a multistage decision making problem under uncertainty [109].

Generically, a $(k+1)$-stage decision making problem (see, e.g., [110]) is defined by a (finite, in practice) time horizon, i.e. an interval $[0 ; T]$ together with a number of time points $t_{0}=0<\ldots<t_{k}=T$, such that:

- the system to be controlled is viewed as a dynamic process $x_{t_{i+1}}=$ $f\left(x_{t_{i}}, u_{t_{i}}, \xi_{t_{i}}\right)$, where $x_{t}$ denotes the system state at some stage, $u_{t}$ denotes the control input (or decision) at this stage, and $\xi_{t}$ represents an uncontrollable and exogenous disturbance process making uncertain the trajectory of the system starting from a known initial state;

- we assume that the initial state $x_{0}$ is known beforehand to the decision maker, and that at each subsequent stage $t_{i}$ the system state $x_{t_{i}}$ together with the history of previous disturbances $\xi_{0}, \ldots, \xi_{t_{i-1}}$ become also perfectly known (i.e. $\xi_{t_{i}}$ is observed only once the decision $u_{t_{i}}$ has been committed);

- we also assume that the decision maker is allowed to choose control sequences $\left[u_{0}, \ldots, u_{t_{k}}\right]$ belonging to a set $U^{*}$ of possible controls (typically constrained in a dynamical, state and disturbance dependent fashion);

- the objective of the decision maker is to determine a control strategy $\left[u_{0}, \ldots, u_{t_{i}}\left(x_{t_{i}}, \xi_{0}, \ldots, \xi_{t_{i-1}}\right), \ldots\right] \in U^{*}$ in order to minimize some cost function depending on the resulting set of possible trajectories induced by the disturbance process, and possibly including hard constraints on the system trajectory.

The classical SCOPF formulation fits in this framework: it uses only two stages, the uncertain process is defined by a finite set of possible contingencies, the objective function depends only on the preventive control $u_{t_{0}}$ and hard security constraints are imposed on $x_{t_{0}}$ and $x_{t_{1}}$. The 3 -stage operation planning problem presented in [111] fits also in this framework: here $u_{t_{0}}$ corresponds to the day-ahead decision while $u_{t_{1}}$ and $u_{t_{2}}$ correspond respectively 
to the next-day's preventive and corrective controls (see also Section 5.4, below).

In the following two subsections we elaborate on two complementary approaches already proposed in the optimal control and stochastic programming literatures to address the generic multistage decision making problem under uncertainty. We believe that these two frameworks should be further developed in order to extend SCOPF technology to the current challenges faced by power system engineers in charge of security management and control.

\subsection{The multistage stochastic programming approach}

The deterministic security criterion used in the conventional SCOPF to handle contingencies exhibits three drawbacks:

1. it treats all contingencies as equiprobable disregarding their individual likelihood of occurrence;

2. it optimizes only the cost of the pre-contingency state controls disregarding the cost of post-contingency corrective actions, assuming thereby that the likelihood of their use is small and that on the long run their cost will remain negligible;

3. it also does not model the costs social and economic of brown-outs and blackouts that may result from the failure of corrective actions. Since such costs are much bigger than the cost of usual corrective actions the question whether this assumption is acceptable remains problematic [116].

Consequently the conventional SCOPF formulation has been questioned by several authors who argue that, in order to mitigate these drawbacks, a stochastic security criterion should be adopted [46, 52, 56, 109, 112, 113, $114,115,116]$. They propose an objective function which trades-off the cost of preventive actions and the expected cost of corrective actions, which we write in the following way:

$$
\min _{\mathbf{x}_{0}, \ldots, \mathbf{x}_{c}, \mathbf{u}_{0}, \ldots, \mathbf{u}_{c}}\left[f_{0}\left(\mathbf{x}_{0}, \mathbf{u}_{0}\right)+\lambda \sum_{k=1}^{c} p_{k} f_{k}\left(\mathbf{x}_{k}, \mathbf{u}_{0}, \mathbf{u}_{k}\right)\right],
$$

where $p_{k}$ is the "probability" of contingency $k$ (with $\sum_{k=1}^{c} p_{k}=1$ ), $f_{k}\left(\mathbf{x}_{k}, \mathbf{u}_{0}, \mathbf{u}_{k}\right.$ ) is the cost of corrective actions and/or constraint violations in the state $k$ induced by contingency $k$, and $\lambda \in[0, \infty$ [ is a parameter defining the tradeoff between costs incurred in preventive and corrective control modes. Notice 
that, for the notational simplicity, all hard constraints are here abstracted away in the cost-functions of equation (14), and also blackout/brownout costs that would be explicitly modeled would be incorporated in the functions $f_{k}$. In this setting, $\lambda$ measures the risk taken by the decision maker, small values corresponding to a risk-prone behavior while large values correspond to a risk-adverse behaviors.

As any approach, the stochastic SCOPF formulation also has some limitations:

1. its output relies on the probabilities of disturbance occurrence for which sufficiently accurate values may not be available. Moreover, the probability of a contingency may depend on the circumstances (e.g. adverse weather conditions or terrorist threat).

2. while the cost of preventive actions is rather easy to calculate, getting a reliable estimate of the cost of corrective actions is a challenging problem due to the difficulty to anticipate the TSO behavior, especially in severe cases (e.g. cascading thermal overloads, voltage unstable scenarios).

3. in order to extend this approach to more than two decision making stages and to cover uncertainties about power system injections, further research on the algorithmic side is needed, in particular to discretize the set of uncertain scenarios in order to build tractable approximations of the mother problem $[110]^{5}$.

However, we believe that the development of appropriate methods to cope with such stochastic multistage programming approaches in the context of security control are a promising avenue for future research. Indeed, recent progress in the fields of approximate stochastic dynamic programming, Monte-Carlo simulation, non-convex optimization, and parallel computation could be exploited to construct credible and tractable approximations for these problems.

\footnotetext{
${ }^{5}$ Note that the solution of the basic stochastic SCOPF problem requires keeping all postulated contingencies in the model as in the direct approach (because the cost of corrective actions intervenes in the objective function). This makes the size of this problem already intractable for very large systems and hence, even for this basic problem, approximations will be required.
} 


\subsection{Robust (min-max) worst case optimal control approach}

We describe hereafter a new proposal for day-ahead operational planning (see e.g. $[17,111]$ ) that avoids the use of probabilistic concepts and substitutes them by concepts from robust control theory.

This approach applies mainly in day-ahead planning, where the focus is on ascertaining the security of the system by looking whether in the worst cases (very extreme patterns of uncertain parameters and contingencies that could show up over the next day) the operator will still have sufficient controllability of the system by combinations of "conventional" preventive/corrective actions (i.e. which can be identified and implemented a few tens of minutes before the real-time e.g. generation re-scheduling, or in real-time after the inception of a contingency e.g. network switching, generation re-scheduling, etc.). The concept is a "look-ahead" preventive security assessment dealing with uncertainty, aimed at determining whether strategic day-ahead planning decisions (e.g. starting up additional units for the next day, or postponing/accelerating foreseen maintenance of some equipments), which generally have to be launched several hours before real time operation, should be taken.

The aim of the worst-case approach is to determine whether, for the assumed range of the uncertain parameters, it is necessary to take such strategic decisions to ensure system controllability over the course of the next day. Such decisions do not have to be taken on the day ahead but should rather be re-assessed periodically, at least until the last moment when such decisions must be implemented, so as to take advantage of the decreasing amount of uncertainty as time progresses.

The principle of the worst-case approach can be summarized as follows: choose day-ahead strategic decisions $\mathbf{u}_{p}$ such that, for any next-day power injection scenario $\mathbf{i}$ (in the assumed uncertainty set) the best combination of conventional preventive actions $\mathbf{u}_{0}$ and corrective actions $\mathbf{u}_{k}$ leads to an acceptable system performance if contingency $k$ occurs.

We define the worst-case operating scenario for a given contingency $k$ as the scenario leading to the largest total violation of post-contingency constraints (either overloads, or voltage limit violations) in the presence of the best possible combination of preventive and corrective actions. Formally this 
leads to the following bi-level optimization problem:

$$
\begin{array}{ll}
\max _{\mathbf{i}} & \mathbf{1}^{T} \boldsymbol{\delta} \\
\text { s.t. } \mathbf{i}^{\min } \leq \mathbf{i} \leq \mathbf{i}^{\max } & \\
\boldsymbol{\delta} \leq \boldsymbol{\delta}_{k}^{\star} & \\
\left(\boldsymbol{\delta}_{k}^{\star}, \mathbf{u}_{0}^{\star}, \mathbf{u}_{k}^{\star}\right)=\arg \quad \min \mathbf{1}^{T} \boldsymbol{\delta}_{k} \\
\text { s.t. } & \mathbf{g}_{0}\left(\mathbf{x}_{0}, \mathbf{u}_{0}, \mathbf{i}\right)=\mathbf{0} \\
& \mathbf{h}_{0}\left(\mathbf{x}_{0}, \mathbf{u}_{0}, \mathbf{i}\right) \leq \mathbf{0} \\
& \mathbf{g}_{k}\left(\mathbf{x}_{k}, \mathbf{u}_{0}, \mathbf{u}_{k}, \mathbf{i}\right)=\mathbf{0} \\
& \mathbf{h}_{k}\left(\mathbf{x}_{k}, \mathbf{u}_{0}, \mathbf{u}_{k}, \mathbf{i}\right) \leq \boldsymbol{\delta}_{k} \\
& \left|\mathbf{u}_{0}-\overline{\mathbf{u}}_{0}\right| \leq \overline{\Delta u}_{0} \\
& \left|\mathbf{u}_{k}-\mathbf{u}_{0}\right| \leq \overline{\mathbf{u}}_{k} \\
& \boldsymbol{\delta}_{k} \geq \mathbf{0},
\end{array}
$$

where $\overline{\mathbf{u}}_{0}$ is the vector of planned optimal settings of base case controls (e.g. obtained previously by a conventional SCOPF (1)-(8) which satisfies all contingency constraints relative to the most likely operating scenario forecasted for the considered period of time of the next day), $\overline{\Delta \mathbf{u}}_{0}$ (resp. $\overline{\Delta \mathbf{u}}_{k}$ ) are the maximal allowed variation of preventive (resp. corrective) actions, $\boldsymbol{\delta}_{k}$ is a vector of positive relaxation terms of the post-contingency inequality constraints, $\mathbf{i}$ is a vector of uncertain bus active/reactive power injections which may vary between the $\operatorname{limits}^{6} \mathbf{i}^{\text {min }}$ and $\mathbf{i}^{\text {max }}$. In this formulation, the strategic control actions $\mathbf{u}_{p}$ have not been made explicit because they are frozen at this optimization stage.

The solution of this bi-level optimization problem can be interpreted as follows. For each possible continuous value of the operating uncertainty vector $\mathbf{i}$ lying on the domain limited by the constraints (16), the slave problem SCOPF (18)-(25) is solved. Let $\left(\boldsymbol{\delta}_{k}^{\star}, \mathbf{u}_{0}^{\star}, \mathbf{u}_{k}^{\star}\right)$ be its solution and let $\mathbf{1}^{T} \boldsymbol{\delta}_{k}^{\star}$ be

\footnotetext{
${ }^{6}$ In the simplest case, the operation uncertainty can take on the form of a bounded active/reactive power, injected at a set of buses from the internal system and/or the neighbouring systems. Determining realistic bounds for the expected range of variation is crucial to obtain reasonable results, since the values of these bounds impact the outcome of the worst-case problem. The range of uncertainty variation can be determined by using the historical record of the values taken by each uncertain variable (e.g., the output of a wind farm) at a given time of the day.
} 
the minimal value of the constraints violation. If this value is zero it signifies that the current uncertainty pattern does not lead to any constraint violation given the postulated preventive/corrective actions. After considering all possible values of the vector $\mathbf{i}$ satisfying the bounds constraints (16), the worst-case operating scenario corresponds to the uncertainty pattern $\mathbf{i}_{k}^{\star}$ that leads to the largest violation of post-contingency constraints. A value of zero of this objective constitutes a very reassuring information for the TSO.

On the other hand if, for one or for several contingencies, the objective (15) is strictly positive, it means that system security cannot be guaranteed by the sole combination of preventive and corrective controls available for the next day. In this case, it will be necessary to determine an appropriate strategic decision $\mathbf{u}_{p}$, that enhances the system controllability during the next day.

Note that there is currently no theoretically sound algorithm able to solve the worst-case bi-level programming problem (15)-(25) given that it is nonconvex, non-linear, very large scale, and involves both discrete and continuous variables [118], and therefore, to determine the worst uncertainty patterns heuristic approaches must be conceived, or (linear) approximations could be used $^{7}$.

A suggested alternative of the worst-case approach consists in modeling uncertainty in the conventional SCOPF (1)-(8) by considering a pre-defined number of base cases (e.g. ranging from no wind to strong wind) and for each of them the whole list of postulated contingencies [10].

\section{Summary and conclusions}

The contributions of this paper are three-fold:

- it reviews the state of the art of computational solutions of the classical SCOPF problem;

- it identifies the open challenges of this problem and highlights promising approaches to face them;

\footnotetext{
${ }^{7}$ For instance, there exists an interesting theoretical result which allows to transform the linearization of this worst-case problem (e.g. under dc approximation assumptions) into a MILP problem [117], for which very powerful solution methods exist.
} 
- it pinpoints some limitations of the classical formulation and indicates possible future research directions in stochastic and robust approaches for multistage decision making under uncertainty that might help overcome these limitations.

The main challenges of the SCOPF problem can be summarized as follows:

1. Problem formulation: some improvements of the SCOPF formulation that should be implemented to obtain solutions that are more realistic and thus more usable by system operators. These improvements are the use of a pre-specified limited number of corrective actions and the handling of transient and voltage stability constraints. Dealing with these makes the problem more complex because it adds new binary variables, it increases significantly the size of the problem, and the SCOPF must be coupled with dynamic simulation to validate the combined optimal preventive and corrective actions. Because the SCOPF is already large and complex, it appears more reasonable to handle transient and voltage stability by adding to the SCOPF linear heuristic constraints derived from dynamic simulation. This would, however, lead to sub-optimal solutions.

2. Problem solution techniques: the following key issues have been discussed:

- reducing the size of the SCOPF problem by efficiently identifying binding contingencies to be included in the SCOPF. However, if the size of the reduced problem is still intractable by actual computers, one may have to rely on approximate models for the post-contingency states.

- treatment of discrete variables

Several mature and sophisticated heuristics approaches exist for handling discrete variables with small discrete steps. At worst the simple, fast, but "blind" round-off strategy can be used. However, for a feasible MINLP SCOPF problem, most of these techniques may not be able to propose settings of discrete variables which ensure the SCOPF feasibility with respect to continuous variables.

On the other hand, the handling of discrete variables with large discrete steps is rather complex due to the large nonlinearities they 
often introduce. This calls for simplified techniques followed by a check of the results using the full nonlinear model. Furthermore, to reduce the combinatorial explosion of the SCOPF it is reasonable to use some the discrete variables with large steps (e.g. network switching) only as post-contingency corrective action, which allows parallel computations for each problematic contingency.

- use of efficient solvers

Mature NLP solvers (e.g. interior point algorithms), which converge reliably and fast, can be used to determine the optimal settings of continuous variables in the SCOPF.

With regard to discrete variables, it makes sense to use approaches relying on MILP, another class of optimization techniques that has recently reached maturity.

The outstanding progress on both optimization field and computers power have made possible SCOPF computations on very large systems for dayahead operational planning using the nonlinear ac network model. Nevertheless, the application of SCOPF in real-time still does not meet the speed requirements for very large systems, unless a dc model is used. Therefore, simplified SCOPF models should be used while seeking to obtain a feasible solution with respect to a maximal subset of contingencies, ignoring less probable contingencies. To speed-up the SCOPF solution, the parallelization of computations can be easily envisaged, where the SCOPF problem is decomposed and distributed among several processors, each one solving independently a limited subset of post-contingency states [43, 119]. Another desirable property of a SCOPF for real-time applications is to be able to efficiently use a hot start in order to take advantage or the previous solution [120].

Future developments in the field of SCOPF will mainly aim to enlarge the scope of this application. They can be summarized as follows:

- Framing the problem as a multistage decision making problem under uncertainty, will make it possible to take advantage of the significant recent progresses in stochastic dynamic programming and robust control approaches, and suggests many interesting directions of research. In particular, the worst-case approach extends the applicability of the SCOPF to the increasingly uncertain day-ahead operational planning context. Stochastic SCOPF formulations have only been solved for 
small test systems and have not yet been accepted by utilities. Furthermore, the problem cannot be decomposed because the cost of postcontingency corrective actions appear in the objective function, and therefore for very large systems simplified approaches may be required.

- The coupling of SCOPF with time domain simulation is another direction of future research in order to ensure that the set of optimal actions proposed by SCOPF do not lead to system instability. Preliminary encouraging results have been obtained [25].

- The computational power of current computers allows us to envisage the inclusion of the SCOPF within the security constrained unit commitment problem, encouraging results being reported [57].

\section{Acknowledgments}

The authors acknowledge their funding by the FP7 EC project PEGASE funded by the European Commission.

F. Capitanescu and L. Wehenkel acknowledge the support of the Belgian Network DYSCO, funded by the Interuniversity Attraction Poles Programme, initiated by the Belgian State, Science Policy Office. The scientific responsibility rests with the authors.

We thank our colleagues M. Ortega-Vazquez, H. Crisciu, Y. Hassaine, and S. Fliscounakis, involved in the WP3 of PEGASE project for their valuable comments.

\section{References}

[1] O. Alsac, B. Stott, Optimal load flow with steady-state security, IEEE Trans. on PAS. 93 (3) (1974) 745-751.

[2] A.J. Monticelli, M.V.P. Pereira, S. Granville, Security-constrained optimal power flow with post-contingency corrective rescheduling, IEEE Trans. Power Syst. 2 (1) (1987) 175-182.

[3] J. Carpentier, Contribution à l'étude du dispatching économique, Bulletin de la Societé Française d'Electricité. (3) 1962 431-447.

[4] H.W. Dommel, W.F. Tinney, Optimal power flow solutions, IEEE Trans. on PAS. 87 (10) (1968) 1866-1876. 
[5] B. Stott, O. Alsac, A.J. Monticelli, Security analysis and optimization (Invited Paper), Proc. of IEEE. 75 (12) (1987) 1623-1644.

[6] A. Papalexopoulos, Challenges to On-Line OPF Implementation, IEEE/PES Winter Meeting, New York (USA), 1995.

[7] S.M. Shahidehpour et al., Nonlinear programming algorithms and decomposition strategies for optimal power flow, IEEE Tutorial, Optimal Power Flow: solution techniques, requirements and challenges, 1996.

[8] J.A. Momoh, R.J. Koessler, M.S. Bond, B. Stott, D. Sun, A. Papalexopoulos, P. Ristanovic, Challenges to optimal power flow, IEEE Trans. on Power Syst. 12 (1) (1997) 444-455.

[9] M. Ilic, AC OPF and smart grids, presentation at FERC Conference on Enhanced Optimal Power Flow Models, Washington, USA, 2010.

[10] R. Zimmeman, A superOPF framework, presentation at FERC Conference on Enhanced Optimal Power Flow Models, Washington, USA, 2010 .

[11] T.J. Overbye, Xu Cheng, Yan Sun, A comparison of the AC and DC power flow models for LMP calculations, Proc. of the 37th Annual HICSS conference, Hawaii, 2004.

[12] Pan European grid advanced simulation and state estimation (PEGASE) project, http://www.fp7-pegase.eu/, 2008.

[13] P. Panciatici (task leader), D. Kirschen, M. Ortega-Vazquez, L. Wehenkel, F. Capitanescu, Deliverable D1.1: Specification and requirements for the European transmission network state estimation and simulation functions, PEGASE project, http://www.fp7-pegase.eu/, 2009.

[14] B. Delourme, A. Lasnier, H. Lefevbre, G. Simeant, Minimizing the cost of generation redispatching taking into account remedial actions, paper C2-103, CIGRE conference, France, 2006.

[15] F. Capitanescu, L. Wehenkel, Improving the statement of the corrective security-constrained optimal power flow problem, IEEE Trans. on Power Syst. 22 (2) (2007) 887-889. 
[16] R. Bacher (Editors: K. Frauendorfer, H. Glavitsch, R. Bacher), Power system models, objectives and constraints in optimal power flow calculations (chapter of the book: Optimization in Planning and Operation of Electric Power Systems), Physica Verlag (Springer), Heidelberg, Germany, 1993, pp. 217-264.

[17] L. Wehenkel (task leader), F. Capitanescu, D. Kirschen, M. OrtegaVazquez, P. Panciatici, S. Fliscounakis, Y. Hassaine, J.L. Martinez Ramos, A. Marano Marcolini, H. Crisciu, L. Platbrood, Deliverable D3.1 (part I): European transmission network modeling requirements for steady state operating conditions with OPF, PEGASE project, http://www.fp7-pegase.eu/, 2009.

[18] W.F. Tinney, J.M. Bright, K.D. Demaree, B.A. Hughes, Some deficiencies in Optimal Power Flow, IEEE Trans. on Power Syst. 3 (1988) 676-683.

[19] S.A. Soman, K. Parthasarathy, D. Thukaram, Curtailed number and reduced controller movement optimization algorithms for real time voltage/reactive power control, IEEE Trans. Power Syst. 9 (4) (1994) 20352041.

[20] W.-H. Edwin Liu, X. Gupa, Fuzzy constraint enforcement and control action curtailment in an optimal power flow, IEEE Trans. Power Syst. 11 (2) (1996) 639-645.

[21] F. Capitanescu, W. Rosehart, L. Wehenkel, Optimal power flow computations with constraints limiting the number of control actions, Power Tech Conference, Bucharest (Romania), 2009.

[22] E.B. Fisher, R.P. O’Neill, M.C. Ferris, Optimal transmission switching, IEEE Trans. Power Syst. 23 (3) (2008) 1346-1355.

[23] F. Capitanescu, L. Wehenkel, Otimal power flow computations with a limited number of controls allowed to move, IEEE Trans. Power Syst. 25 (1) (2010) 586-587.

[24] F. Capitanescu, L. Wehenkel, Redispatching active and reactive powers using a limited number of control actions, IEEE Trans. Power Syst. in press, DOI 10.1109/TPWRS.2010.21023712011, 2011. 
[25] F. Capitanescu, T. Van Cutsem, L. Wehenkel, Coupling optimization and dynamic simulation for preventive-corrective control of voltage instability, IEEE Trans. Power Syst. 24 (2) (2009) 796-805.

[26] E. Vaahedi, H.M. Zein El-Din, Considerations in applying optimal power flow to power system operation, IEEE Trans. on Power Syst. 4 (2) (1989) 694-703.

[27] D. Gan, R.J. Thomas, R.D. Zimmerman, A Transient Stability Constrained Optimal Power Flow, Proc. Bulk Power System Dynamics and Control IV - Restructuring, Santorini (Greece), 1998.

[28] M. La Scala, M. Trovato, C. Antonelli, On-line Dynamic Preventive Control: An Algorithm for Transient Security Dispatch, IEEE Trans. Power Syst. 13 (2) (1998) 601-610.

[29] S. Bruno, E. De Tuglie, M. La Scala, Transient Security Dispatch for the Concurrent Optimization of Plural Postulated Contingencies, IEEE Trans. on Power Syst. 17 (3) (2002) 707-714.

[30] Y. Yuan, J. Kubokawa, H. Sasaki, A Solution of Optimal Power Flow with Multicontingency Transient Stability Constraints, IEEE Trans. Power Syst. 18 (3) (2003) 1094-1102.

[31] R. Zarate-Minano, T. Van Cutsem, F. Milano, A.J. Conejo, Securing Transient Stability Using Time-Domain Simulations Within an Optimal Power Flow, IEEE Trans. on Power Syst. 25 (1) (2010) 243-253.

[32] A. Pizano-Martianez, C.R. Fuerte-Esquivel, D. Ruiz-Vega, Global Transient Stability-Constrained Optimal Power Flow Using an OMIB Reference Trajectory, IEEE Trans. on Power Syst. 25 (1) (2010) 392-403.

[33] D. Ruiz-Vega, M. Pavella, A comprehensive approach to transient stability control: part 1 - near optimal preventive control, IEEE Trans. on Power Syst. 18 (4) (2003) 1446-1453.

[34] E. De Tuglie, M. La Scala, P. Scarpellini, Real-time preventive actions for the enhancement of voltage-degraded trajectories, IEEE Trans. Power Syst. 14 (2) (1999) 561-568. 
[35] E. De Tuglie, M. Dicorato, M. La Scala, P. Scarpellini, A corrective control for angle and voltage stability enhancement on the transient time-scale, IEEE Trans. Power Syst. 15 (4) (2000) 1345-1353.

[36] E. Vaahedi, Y. Mansour, C. Fuchs, S. Granville, M. de Lujan Latore, H. Hamadanizadeh, Dynamic Security Constrained Optimal Power Flow / VAR Planning, IEEE Trans. Power Syst. 16 (1) (2001) 38-43.

[37] F. Milano, C. A. Canizares, M. Ivernizzi, Voltage Stability Constrained OPF Market Models Considering N-1 Contingency Criteria, Electric Power Systems Research. 74 (1) (2005) 27-36.

[38] H. Song, B. Lee, S.H. Kwon, V. Ajjarapu, Reactive reserve-based contingency constrained optimal power flow (RCCOPF) for enhancement of voltage stability margins, IEEE Trans. Power Syst. 18 (4) (2003) 1538-1546.

[39] F. Capitanescu, T. Van Cutsem, Preventive control of voltage security margins: a multi-contingency sensitivity-based approach, IEEE Trans. Power Syst. 17 (2) (2002) 358-364.

[40] J.L. Martinez Ramos (task leader), A. Marano Marcolini, F. Capitanescu, L. Wehenkel, D. Kirschen, M. Ortega-Vazquez, P. Panciatici, S. Fliscounakis, Y. Hassaine, H. Crisciu, L. Platbrood, Deliverable D3.1 (part II): Description of the state of the art in OPF and the requirements for European transmission network optimization problems, PEGASE project, http://www.fp7-pegase.eu/, 2009.

[41] O. Alsac, J. Bright, M. Prais, B. Stott, Further developments in LPbased optimal power flow, IEEE Trans. Power Syst. 5 (3) (1990) 697711.

[42] T.J. Bertram, K.D. Demaree, L.C. Dangelmaier, An integrated package for real-time security enhancement, IEEE Trans. Power Syst. 5 (2) (1990) 592-600.

[43] M. Rodrigues, O.R. Saavedra, A. Monticelli, Asynchronous programming model for the concurrent solution of the security constrained optimal power flow problem, IEEE Trans. Power Syst. 9 (4) (1994) 20212027. 
[44] F. Capitanescu, M. Glavic, D. Ernst, L. Wehenkel, Contingency filtering techniques for preventive security-constrained optimal power flow, IEEE Trans. Power Syst. 22 (4) (2007) 1690-1697.

[45] F. Capitanescu, L. Wehenkel, A new iterative approach to the Corrective Security-Constrained Optimal Power Flow Problem, IEEE Trans. on Power Syst. 23 (4) (2008) 1533-1541.

[46] F. Bouffard, F.D. Galiana, J.M. Arroyo, Umbrella contingencies in security-constrained optimal power flow, Proc. of the 15-th Power Systems Computation Conference (PSCC), Liège (Belgium), 2005.

[47] L. Platbrood (task leader), H. Crisciu, C. Merckx, P. Panciatici, S. Fliscounakis, F. Capitanescu, L. Wehenkel, D. Kirschen, M. OrtegaVazquez, Deliverable D3.3: Prototypes for European transmission system steady-state optimisation, PEGASE project, http://www.fp7pegase.eu/, 2011.

[48] J.F. Benders, Partitioning procedure for solving mixed variables programming problems, Numerische Mathematics, 4 (1962) 238-252.

[49] A.M. Geoffrion, Generalized Benders decomposition, Journal of Optimization Theory and Applications, 10 (4) (1972) 237-260.

[50] T. Gomez, I.J. Perez-Arriaga, J. Lumbreras, V.M. Parra, A securityconstrained decomposition approach to optimal reactive power planning, IEEE Trans. Power Syst. 6 (3) (1991) 1069-1076.

[51] S. Granville, M.C. Abib Lima, Application of decomposition techniques to VAr planning: methodological and computational aspects, IEEE Trans. Power Syst. 9 (4) (1994) 1780-1787.

[52] G. Strbac, S. Ahmed, D. Kirschen, R. Allan, A method for computing the value of corrective security, IEEE Trans. Power Syst. 13 (3) (1998) 1096-1102.

[53] R.A. Schlueter, S. Liu, N. Alemadi, Preventive and corrective open access system dispatch based on the voltage stability security assessment and diagnosis, Electric Power Syst. Research, 60 (2001) 17-28. 
[54] W. Li, M. Shaaban, Z. Yan, Y. Ni, F.F. Wu, Available transfer capability calculation with static security constraints, IEEE PES General Meeting, (2003) 306-310.

[55] Yuan Li, J.D. McCalley, Decomposed SCOPF for Improving Efficiency, IEEE Trans. on Power Syst. 24 (1) (2009) 494-495.

[56] F. Bouffard, F.D. Galiana, A.J. Conejo, Market-clearing with stochastic security (parts I and II), IEEE Trans. Power Syst. 20 (4) (2005) 18181835.

[57] J. Martinez-Crespo, J. Usaola, J.L. Fernandez, Security-constrained optimal generation scheduling in large-scale power systems, IEEE Trans. Power Syst. 21 (1) (2006) 321-332.

[58] Y. Fu, M. Shahidehpour, Z. Li, AC contingency dispatch based on security-constrained unit commitment, IEEE Trans. Power Syst. 21 (2) (2006) 897-908.

[59] J.L. Martinez Ramos, V.H. Quintana, Optimal and Secure Operation of Transmission Systems, Electric Energy Systems, E. Gomez, A. Conejo, C. Canizares (Eds.), CRC Press, 2009, pp. 211-264.

[60] R. A. Jabr, A. H. Coonick, B. J. Cory, A homogeneous linear programming algorithm for the security constrained economic dispatch problem, IEEE Trans. Power Syst. 15 (3) (2000) 930-936.

[61] D. Kirschen, H.P. Van Meeteren, MW voltage control in a linearprogramming based optimal power flow, IEEE Trans. Power Syst. 3 (3) (1988) 481-489.

[62] K.W. Hedman, R.P. O'Neill, E.B. Fisher, S.S. Oren, Optimal transmission switching with contingency analysis, IEEE Trans. Power Syst. 24 (3) (2009) 1577-1586.

[63] K. Karoui, H. Crisciu, A. Szekut, M. Stubbe, Large scale security constrained optimal power flow, Proc. of the 16-th Power Systems Computation Conference (PSCC), Glasgow (Scotland), 2008.

[64] Y.C. Wu, A.S. Debs, R.E. Marsten, A Direct Nonlinear PredictorCorrector Primal-Dual Interior Point Algorithm for Optimal Power Flows, IEEE Trans. Power Syst. 9 (2) (1994) 876-883. 
[65] H. Wei, H. Sasaki, J. Kubokawa, R. Yokohama, An Interior Point Nonlinear Programming for Optimal Power Flow with a Novel Data Structure, IEEE Trans. Power Syst. 13 (3) (1998) 870-877.

[66] S. Granville, Optimal reactive dispatch through interior point methods, IEEE Trans. Power Syst. 9 (1) (1994) 136-146.

[67] S. Granville, J.C.O. Mello, A.C.G. Melo, Application of interior point methods to power flow unsolvability, IEEE Trans. Power Syst. 11 (4) (1996) 1096-1103.

[68] G.D. Irrisari, X. Wang, J. Tong, S. Mokhtari, Maximum loadability of power systems using interior point nonlinear optimization methods, IEEE Trans. Power Syst. 12 (1) (1997) 162-172.

[69] G.L. Torres, V.H. Quintana, An Interior-Point Method for Nonlinear Optimal Power Flow Using Rectangular Coordinates, IEEE Trans. Power Syst. 13 (4) (1998) 1211-1218.

[70] G.L. Torres, V.H. Quintana, On a Nonlinear Multiple-CentralityCorrections Interior-Point Method for Optimal Power Flow, IEEE Trans. Power Syst. 16 (2) (2001) 222-228.

[71] W. Min, L. Shengsong, A trust region interior point algorithm for optimal power flow problems, International Journal of Electrical Power and Energy Systems, 24 (4) (2005) 293-300.

[72] A.A. Sousa, G.L. Torres, C. Canizares Robust Optimal Power Flow Solution Using Trust Region and Interior-Point Methods, IEEE Trans. Power Syst., 26 (2) (2011) 487-499.

[73] K. Karoui, L. Platbrood, H. Crisciu, R.A. Waltz, New large-scale security constrained optimal power flow program using a new interior-point algorithm, 5-th International Conference on European Electricity Market, 2008 .

[74] H. Wang, C.E. Murillo-Sanchez, R. Zimmerman, R.J. Thomas, On computational issues of market-based optimal power flow, IEEE Trans. Power Syst. 22 (3) (2007) 1185-1192. 
[75] KNITRO solver description, available on-line at: http://www.ziena.com/knitro.htm.

[76] A. Wachter, L. T. Biegler, On the Implementation of a Primal-Dual Interior Point Filter Line Search Algorithm for Large-Scale Nonlinear Programming, Mathematical Programming, 106 (1) (2006) 25-57.

[77] D.F. Shanno, R.J. Vanderbei, Interior-Point Methods for Nonconvex Nonlinear Programming: Orderings and Higher-Order Methods, Math. Prog. 87 (2) (2000) 303-316.

[78] A.D. Papalexopoulos, C.F. Imparato, F.W. Wu, Large-Scale Optimal Power Flow: Effects of Initialization, Decoupling \& Discretization, IEEE Trans. Power Syst. 4 (1989) 748-759.

[79] E. Liu, A.D. Papalexopoulos, W.F. Tinney, Discrete Shunt controls in a Newton Optimal Power Flow, IEEE Trans. Power Syst. 7 (1992) 15191528.

[80] M. Liu, S.K. Tso, Y. Cheng, An Extended Nonlinear Primal-Dual Interior-Point Algorithm for Reactive Power Optimization of LargeScale Power Systems with Discrete Control Variables, IEEE Trans. Power Syst. 17 (4) (2002) 982-991.

[81] S.Y. Lin, Y.C. Ho, C.H. Lin, An Ordinal Optimization Theory-Based Algorithm for Solving Optimal Power Flow Problem with Discrete Control Variables, IEEE Trans. Power Syst. 19 (1) (2004) 276-286.

[82] K. Aoki, M. Fan, A. Nishikori, Optimal var planning by approximation method for recursive mixed-integer linear programming, IEEE Trans. on Power Syst. 3 (4) (1988) 1741-1747.

[83] X. Ding, X. Wang, Y.H. Song, Interior point cutting plane method for optimal power flow, IMA Journal of management mathematics, 15 (4) (2004) 355-368.

[84] L. Liu, X. Wang, X. Ding, H. Chen, A Robust Approach to Optimal Power Flow With Discrete Variables, IEEE Trans. Power Syst. 24 (3) (2009) 1182-1190. 
[85] F. Capitanescu, L. Wehenkel, Sensitivity-based approaches for handling discrete variables in optimal power flow computations, IEEE Trans. Power Syst. 25 (4) (2010) 1780-1789.

[86] A.G. Bakirtzis, P.N. Biskas, C.E. Zoumas, V. Petridis, Optimal Power Flow by Enhanced Genetic Algorithm, IEEE Trans. Power Syst. 17 (1) (2002) 229-236.

[87] L. Chen, H. Suzuki, K. Katou, Mean-field theory for optimal power flow, IEEE Trans. Power Syst. 12 (4) 1997 1481-1486.

[88] T. Kulworawanichpong, S. Sujitjorn, Optimal Power Flow using tabu search, IEEE Power Engineering review, (2002), 37-40.

[89] W. Yan, F. Liu, C.Y. Chung, K.P. Wong, A Hybrid Genetic AlgorithmInterior Point Method for Optimal Reactive Power Flow, IEEE Trans. Power Syst. 21 (3) (2006) 1163-1169.

[90] H. Koglin, H. Muller, Overload reduction through corrective switching actions, Proc. of Int. Conf. on Power Syst. Monit. and Control, 187 (1980) 159-164.

[91] R.A.M. Van Amerongen, H.P. Van Meeteren, Security Control by Real Power Rescheduling, Network Switching and Load Shedding, CIGRE Report 32-02, France, 1980.

[92] R. Bacher, H. Glavitsch, Network Topology Optimization With Security Constraints, IEEE Trans. Power Syst. 1 (4) (1986) 103-111.

[93] A. Mazi, B.F. Wollenberg, M.H. Hesse, Corrective control of power system flow by line and bus-bar switching, IEEE Trans. Power Syst. 1 (3) (1986) 258-265.

[94] J. N. Wrubel, P.S. Rapcienski, K.L. Lee, B.B. Gisin, G.W. Woodrell, Practical experience with corrective switching algorithm for on-line applications, IEEE Trans. Power Syst. 12 (1) (1996) 415-421.

[95] E. Lobato, F. Echavarren, L. Rouco, M.I. Navarrete, R. Casanova, G. Lopez, A Mixed-Integer LP Based Network Topology Optimization Algorithm for Overload Alleviation, PowerTech Conference, Bologna (Italy), 2003. 
[96] W. Shao, V. Vittal, Corrective switching algorithm for relieving overloads and voltage violations, IEEE Trans. Power Syst. vol. 20 (4) (2005) 1877-1885.

[97] G. Granelli, M. Montagna, F. Zanellini, P. Bresesti, R. Vailati, M. Innorta, Optimal network reconfiguration for congestion management by deterministic and genetic algorithms, Electr. Power Syst. Res. 76 (2006) 549-556.

[98] A.G. Bakirtzis, A.P. Meliopoulos, Incorporation of switching operations in power system corrective control computations, IEEE Trans. ower Syst. 2 (3) (1987) 669-676.

[99] J.G. Rolim, L.J.B. Machado, A study of the use of corrective switching in transmission systems, IEEE Trans. Power Syst. 14 (1) (1999) 336-341.

[100] C.A. Rossier, A. Germond, Network Topology Optimization for Power System Security Enhancement, CIGRE IFAC Symp. Control Application for Power System Security, Florence (Italy), 1983.

[101] G. Schnyder, H. Glavitsch, Security Enhancement Using An Optimal Switching Power Flow, IEEE Trans. Power Syst. 5 (2) (1990) 674-681.

[102] R. Bacher, H. Glavitsch, Loss reduction by network switching, IEEE Trans. Power Syst. 3 (2) (1988) 447-454.

[103] S. Fliscounakis, F. Zaoui, M.P. Houry, E. Milin, Loss reduction as a Mixed Integer optimization problem, IEEE PES PowerTech conference, Bucharest (Romania), 2009.

[104] H. Glavitsch, State of the Art Review - Switching as means of control in the power system, International Journal of Electrical Power and Energy Systems, 7 (2) (1985) 92-100.

[105] E. Lobato, L. Rouco, T. Gomez, F. Echavarren, M. Navarrete, R. Casanova, G. Lopez, A Practical Approach to Solve Power System Constraints With Application to the Spanish Electricity Market, IEEE Trans. Power Syst. 19 (4) (2004) 2029-2037.

[106] H. Pinto, F. Magnago, S. Brignone, O. Alsac, B. Stott, Security constrained unit commitment: network modeling and solution issues, IEEE PSCE conference, (2006) 1759-1766. 
[107] K. Zhou, J. C. Doyle, K. Glover, Robust and optimal control, Pretice Hall, New Jersey, 1995.

[108] J.R. Birge, F. Louveaux, Introduction to stochastic programming, Springer-Verlag, Berlin, 1997.

[109] L. Wehenkel, Emergency control and its strategies (invited paper), Proc. of PSCC conference, Trondheim (Norway), 1999, pp. 35-48.

[110] B. Defourny, D. Ernst, L. Wehenkel, Multistage stochastic programming: A scenario tree based approach to planning under uncertainty, To appear in L. E., Sucar, E. F., Morales, and J., Hoey (Eds.), Decision Theory Models for Applications in Artificial Intelligence: Concepts and Solutions. Hershey, Pennsylvania, USA: Information Science Publishing, DOI 10.4018/978-1-60960-165-2, 2011, 51 pages.

[111] P. Panciatici, S. Fliscounakis, Y. Hassaine, J.L. Martinez-Ramos, M. Ortega-Vazquez, L. Platbrood, L. Wehenkel, Security management under uncertainty: from day-ahead planning to intraday operation, IREP Symposium, Buzios (Brazil), 2010.

[112] J. Carpentier, D. Menniti, A. Pinnarelli, N. Scordino, N. Sorrentino, A model for the ISO Insecurity Costs Management in a Deregulated Market Scenario, IEEE PowerTech Conference, Porto (Portugal), 2001.

[113] L.M. Kimball, K.A. Clements, S. Pajic, P.W. Davis, Stochastic OPF by constraint relaxation, IEEE Power Tech Conference, Bologna (Italy), (2003) 23-26.

[114] J. Condren, T.W. Gedra, P. Damrongkulkamjorn, Optimal power flow with expected security costs, IEEE Trans. Power Syst. 21 (2) (2006) 541-547.

[115] Fei Xiao, J.D. McCalley, Risk-Based Security and Economy Tradeoff Analysis for Real-Time Operation, IEEE Trans. Power Syst. 22 (4) (2007) 2287-2288.

[116] M.A. Rios, D. Kirschen, D. Jayaweera, D. Nedic, R. Allan, Value of Security: Modeling Time-Dependent Phenomena and Weather Conditions, IEEE Trans. Power Syst. 17 (3) (2002) 543-548. 
[117] C. Audet, P. Hansen, B. Jaumard, G. Savard, Links between linear bilevel and mixed 0-1 programming problems, Journal of Optimization Theory and Applications, 93 (2) (1997) 273-300.

[118] B. Colson, P. Marcotte, G. Savard, An overview of bilevel optimization, Annals of Operations Research, 1 (2007) 235-256.

[119] W. Qiu, A.J. Flueck, F. Tu, A new parallel algorithm for security constrained optimal power flow with a nonlinear interior-point method, IEEE PES General Meeting, 2005, pp. 447-453.

[120] H. Harsan, N. Hadjsaid, P. Prouvot, Cyclic Security Analysis for Security Constrained Optimal Power Flow, IEEE Trans. Power Syst. 12 (2) (1997) 948-953. 